\title{
Avaliação de um Jogo para o Ensino de Gerência de Riscos: uma Experiência com o Modelo de Avaliação MEEGA+
}

\author{
Rodrigo Cezario da Silva \\ Curso de Sistemas de Informação \\ UNIAVAN - Centro Universitário Avantis \\ Balneário Camboriú, SC, Brasil \\ rodrigo.cezario@uniavan.edu.br
}

\author{
Déryk Frank Rios Fernandes \\ Curso de Sistemas de Informação \\ UNIAVAN - Centro Universitário Avantis \\ Balneário Camboriú, SC, Brasil \\ derykfrank@gmail.com
}

\begin{abstract}
Educational games are used as practical learning tools. However, most of the games do not have evaluations that present learning results that confirm their effectiveness. Therefore, we aimed to evaluate the game The Risk Analysis Theory aimed at the area of risk management knowledge, using the model called MEEGA+ to evaluate the perception of learning and experience of the players. The result of the evaluation presents indications that the game is effective in learning and provides fun to the players guaranteeing their use of the game as a learning tool, however, some points of improvement of the game have been highlighted. This study also presents the result of the evaluation of participants 'level of knowledge about risk management, allowing us to observe that the intervention with gambling provided an increase in participants' knowledge about the topic addressed.
\end{abstract}

\section{KEYWORDS}

Educational game, Software project management, Evaluation, Non- digital games.

\section{Introdução}

A Gerencia de Projetos é uma disciplina presente na maioria dos cursos superiores da área de computação. A disciplina está relacionada à área de Engenharia de Software (ES) que se tornou muito importante pois busca de maneira controlada, metódica e disciplinada a eficiência e qualidade no desenvolvimento de software [1].

A Engenharia de Software tem um papel fundamental e de grandes responsabilidades dentro das organizações, que exigem de um profissional, muitas competências voltadas para a criação do software, por exemplo, conhecimentos de padrões ligados a software, qualidade, capacidade de inovação, criatividade e habilidades interpessoais [2]. Dentre as várias áreas de conhecimento estudadas em ES e que fazem parte da Gerência de Projetos está o Gerenciamento de Riscos que se destaca por apresentar fundamentais ferramentas para tomada de decisão efetiva em resposta para as organizações [3]. Mendes e Oliveira [4] explicam que se estuda a Gerência de Riscos como um dos principais modelos de qualidade de software para se ter um projeto bem-sucedido, pois gerencia a probabilidade de um evento inesperado ocorrer e/ou suas consequências, denominado assim como risco. Ainda segundo Pressman [3], o gerenciamento de riscos tem um papel importante na viabilidade dos planos de projeto, melhorando a busca e identificação de problemas potenciais que podem afetar as atividades do ciclo de vida, a qualidade e o desempenho dos produtos e também para melhorar o gerenciamento ativo dos projetos.

Por sua vez, o ensino da ES aplicado nos cursos superiores enfrenta problemas quanto à aprendizagem durante as aulas, onde, conforme explica Silva [10], muitos alunos não absorvem totalmente o ensino da ES pela fato de possuir conteúdo denso. Todavia, o autor cita que esses problemas vão além das questões teóricas, envolvendo logo, a parte prática. Frente a isto, entendese que para conceituar o real objetivo das áreas de conhecimento de gerência de projetos, é necessário um amplo caminho para que o aluno possa ter uma visão real da aplicação dos processos de gerência de projetos [6]. Portanto, mudar a forma de ensino de Gerência de Projetos buscando e utilizando novas estratégias implica positivamente na qualidade dos profissionais, pois o modelo tradicional de ensino de ES não é apropriado para uma efetiva aprendizagem [1,7].

Com base em estudos de novas formas de ensinoaprendizagem, são então propostos os Jogos Educacionais [6]. Os jogos são de grande importância, possibilitando maior absorção de conhecimentos, bem como despertam a criatividade $\mathrm{e}$ interesse do aluno [8]. Os jogos permeiam ambientes que buscam desenvolver especificamente as habilidades intrínsecas aos alunos através das atividades lúdicas [9]. Consoantes com Paulo e Raabe [9], Fernandes e Werner [10] entendem que os conceitos podem ser melhor captados pelos alunos por meio dos jogos, sem que estes percebam. Sendo assim, o uso de jogos para o ensino de ES pode contribuir significativamente com a transferência de conhecimento de acordo com as metas educacionais [11].

Desta forma, o jogo The Risk Analysis Theory contribui como uma ferramenta de apoio ao ensino de gerenciamento de risco em disciplina de Gerência de Projetos [12]. Segundo Lamin [12], o jogo permite simular atividades do cotidiano da gerência de projetos, agregando conhecimento na área de gerenciamento de risco. O jogo The Risk Analysis Theory é um jogo de tabuleiro cujo seu layout foi baseado na série televisiva chamada de The Big Bang Theory, que remete ao universo geek. No jogo, os jogadores devem aplicar recomendações descritas pelo PMBOK [13] no gerenciamento de riscos, em conjunto com um estudo de caso adotado pelo professor da disciplina. Segundo Lamin [12], o jogo permite aos jogadores exercitarem os conceitos acerca do gerenciamento de risco de projetos em diversos níveis cognitivos da taxonomia de Bloom, como: conhecimento, compreensão, aplicação e análise. A mecânica básica do jogo considera que o 
jogador deve percorrer o tabuleiro acumulando pontos de experiência a partir de situações ou atividades práticas nas quais o jogador deve apresentar conhecimento sobre o gerenciamento de riscos.

Destaca-se que os jogos educacionais são considerados uma estratégia instrucional eficiente para o ensino de computação [6]. Em contraponto, All et al. [15] observam que nem todos os jogos são realmente efetivos como ferramenta de apoio ao ensinoaprendizagem, especialmente os jogos para educação em computação. Todavia é essencial avaliar sistematicamente esses jogos a fim de obter evidências de sua qualidade [16].

No trabalho de Lamin [12], uma avaliação da percepção de uso do jogo foi realizada junto ao grupo de participantes. Apesar de Lamin [12] ter observado que o jogo pode ser utilizado como ferramenta de apoio ao ensino, e de que a utilização do jogo motivou os participantes em conhecer e utilizar os artefatos típicos da gestão de riscos, a avaliação apresenta alguns vieses, como: (i) amostra pequena (apenas 3 participantes) para demonstrar algum resultado significativo; (ii) apesar de planejada, nenhuma avaliação sobre os níveis de conhecimento foi aplicada; (iii) não está claro se a observação de atratividade do jogo aparece no plano do experimento. Neste sentido, o objetivo deste estudo foi de realizar uma avaliação da qualidade do jogo, sendo aplicada em uma turma da disciplina de Gerência de Projetos no sentido de resolver os vieses destacados anteriormente.

\subsection{Justificativa}

Para Jappur et al. [17], os jogos educativos são um aporte para os processos de aprendizagem nas salas de aula como forma de conteúdo. No tocante, Savi e Ulbricht [18] destacam que os jogos educativos trazem como didática benefícios para as práticas de ensino-aprendizagem mais próximas da realidade. Desta forma, Ariffin et al. [19] concluem que os jogos instrucionais ajudam na aprendizagem e educação, pois replicam ações do mundo real através de regras, restrições e atividades. Assim sendo, jogos educacionais mesmo possuindo mais abrangência na aplicação de ensino, ainda carecem de estudos que avaliem sua efetividade para o aprendizado e treinamento [19]. Segundo Petri et al. [16], os jogos educacionais são considerados como estratégia para o ensino, porém eles devem ser avaliados para se ter evidências da qualidade de sua aplicação.

Diante da importância de se avaliar jogos aplicados nos processos de ensino-aprendizagem, o presente estudo apresenta o resultado da avaliação o jogo The Risk Analysis Theory [12] utilizando o modelo MEEGA+ [16]. Além disso, aproveitando a oportunidade, realizou-se uma avaliação para verificar se houve aumento no nível de conhecimento dos participantes. Espera-se com este trabalho contribuir demonstrando a eficácia do jogo avaliado como ferramenta de apoio ao ensino em disciplinas de gerenciamento de riscos.

\section{Uso de Jogos no Ensino de Engenharia de Software}

De acordo com Paludo e Raabe [9], o atual cenário de ensino à Engenharia de Software nos cursos de formação superior, possuem métodos didáticos que pouco contribuem para aplicação prática dos conceitos vistos em sala de aula. Segundo Brito et al. [20], é preciso aperfeiçoar a qualidade do ensino na área de
Engenharia de Software nas universidades para se ter profissionais bem qualificados, desta forma, os jogos educacionais são uma alternativa viável como método de aprendizagem capaz de ensinar os alunos enquanto se divertem.

Os jogos possibilitam a experimentação de vivências fora do contexto educacional, situações estas ligadas diretamente ao ambiente profissional [5]. Consequentemente, por propiciarem práticas educacionais atrativas e inovadoras, em que os alunos podem aprender de forma mais dinâmica e motivadora, os jogos educacionais conseguem ser auxiliares importantes no ensino aprendizagem [18]. De acordo com Monsalve et al. [21], os jogos educacionais para ensino são uma poderosa ferramenta de aprendizagem, simulando ambientes reais, melhorando o desempenho dos alunos e estimulando a geração de experiências individuais, coletivas e sociais, que ajudam na formação de profissionais.

Para o ensino de Engenharia de Software em questão, entende-se que o grande conjunto da grade curricular pode ser extenso e de difícil compreensão de todo o conjunto aplicável de teorias para o alcance da qualidade de software. Meirelles et al. [22] conceituam dificuldades relatada pelos alunos em assimilarem os principais conceitos da Gerência de Projetos, como gerência de pessoas, gerência de riscos, gerência de tempo e controle de versões, pois alguns conceitos teóricos ficam distantes da prática.

Diversos jogos educacionais vendo sendo propostos para utilização no ensino da Engenharia de Software que visam garantir ferramentas de apoio para transmitir conhecimentos $[23,22,14]$. Assim, a qualidade na transmissão de conhecimentos em Engenharia de Software necessita de mudanças, e uma vez evoluída, os estudantes da área, terão maior qualificação profissional e estarão aptos a resolver os problemas relacionados ao desenvolvimento de software, resultando no aumento de projetos bem-sucedidos. Entretanto, surge a dificuldade de instruir o profissional docente de conciliar de forma efetiva as atividades focadas no professor e as focadas no aluno e na disciplina em um curto prazo de tempo e que possa ser aplicado com instrumentos educacionais de fácil alcance para as universidades [20].

Portanto, dentre as vantagens de utilizar jogos educacionais, pode-se destacar a motivação e o aprendizado vivencial e, os jogos devem principalmente transmitir motivação e satisfação aos estudantes [23]. Certamente os jogos podem proporcionar a vivência em experiências de aprendizagem concretas [24].

\subsection{O The Risk Analysis Theory}

O jogo The Risk Analysis Theory é um jogo educativo de Gerenciamento de Riscos em Gerência de Projetos que tem o objetivo de simular os processos encontrados no dia a dia por um profissional da área de gerenciamento de projetos, gerando aprendizado e compreensão baseado nas regras do PMBOK [12]. O objetivo principal do jogo é ajudar os jogadores a lembrar, compreender e aplicar os conceitos acerca do gerenciamento de risco de projetos [12].

Tendo o público alvo do jogo, alunos dos cursos de computação, Lamin [12] escolheu basear a temática do jogo na série de televisão norte-americana de comédia chamada de The Big Bang Theory. No contexto educacional segundo Lamin [12], os jogadores devem aplicar recomendações descritas pelo 
PMBOK [13] no gerenciamento de riscos, aplicados em conjunto com um estudo de caso adotado pelo professor da disciplina. Segundo Lamin [12], o jogo permite os jogadores exercitarem os conceitos acerca do gerenciamento de risco de projetos em diversos níveis cognitivos da taxonomia de Bloom, como: conhecimento, compreensão, aplicação e análise. A partir de uma narrativa simples, que descreve que o jogador (de dois a seis jogadores) deve percorrer o tabuleiro acumulando pontos de experiência a partir de situações ou atividades práticas nas quais o jogador apresenta conhecimento sobre o gerenciamento de riscos.

A mecânica do jogo descreve um tabuleiro (ilustrado na Figura 1) com 6 (seis) áreas distintas em que cada uma delas se refere a um processo de gerenciamento de riscos de projeto (planejar o gerenciamento de riscos, identificar os riscos, realizar análise qualitativa, realizar análise quantitativa, realizar respostas aos riscos e controlar os riscos) sendo delimitadas por cores diferentes onde cada uma apresenta atividades relacionadas a uma área da gerência de riscos.

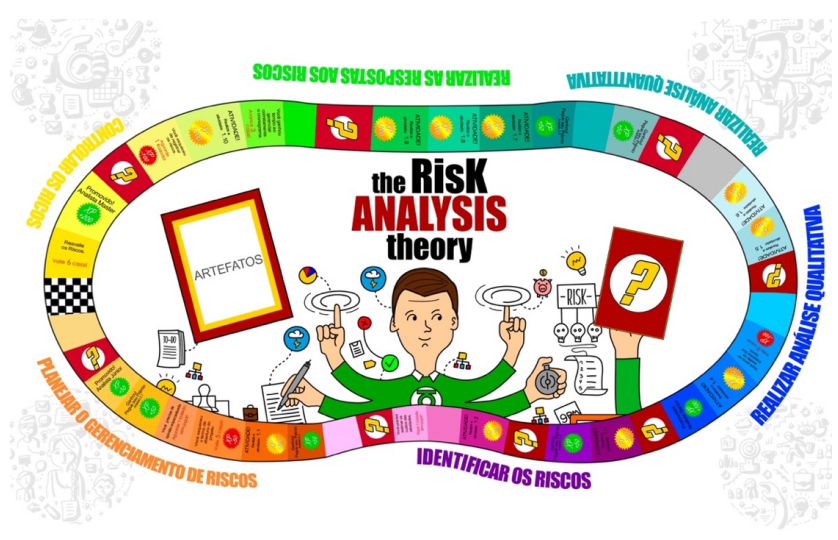

Figura 1: Tabuleiro do jogo The Risk Analysis Theory [12]

A partir do lance de dados, o jogador movimenta seu token o número casas do tabuleiro indicado pelos dados. Ao chegar em uma casa, o jogador receberá instruções para o cumprimento das tarefas que variam entre: avançar casas; voltar um certo número de casas; ficar uma rodada sem jogar; sortear cartas chamadas de cartões de conhecimento, onde são apresentadas boas e má práticas no gerenciamento de riscos; e, executar uma atividade. Ao sortear uma carta, o jogador poderá ganhar ou perder pontos de experiência de acordo com o tipo da prática apresentada (boas práticas acrescentam pontos de experiência e más práticas decrescem pontos de experiência). É importante ressaltar que as cartas apresentam oportunidades de aprendizagem para os jogadores, descrevendo boas e más práticas no gerenciamento de riscos em projetos.

Ao alcançar uma casa relacionada à execução de uma atividade, os demais jogadores deverão entrar em consenso em relação a sua resolução para que o jogador que realizou a atividade ganhe os pontos de experiência referentes à atividade. O Quadro 1 apresenta um exemplo de atividade prática. Lamin [12] observa que as atividades devem ser realizadas considerando o projeto descrito no estudo de caso definido previamente pelo professor da disciplina.
Quadro 1: Exemplo de atividade prática [12]

ATIVIDADE 1.1: IDENTIFIQUE UM RISCO NEGATIVO QUE PODE
AFETAR O PROJETO.

Baseado no estudo de caso, liste um risco que pode afetar de forma negativa o projeto.

$>$ HELP!

Riscos podem ser considerados eventos ou condições incertas que, caso ocorram, provocam um efeito positivo ou negativo nos objetivos do projeto. Os riscos incluem tanto as ameaças aos objetivos do projeto quanto as oportunidades de melhorar o desempenho desses objetivos.

Outro item da mecânica do jogo corresponde a artefatos utilizados no gerenciamento de riscos. Um exemplo de artefato é retratado na Figura 2, onde é apresentada uma matriz de probabilidade e impacto (Artefato 2.3).

\begin{tabular}{|c|c|c|c|c|c|c|c|c|c|c|}
\hline \multicolumn{11}{|c|}{ Matriz de probabilidade e impacto } \\
\hline \multirow{2}{*}{\begin{tabular}{|c|} 
Probabilidade \\
0.90 \\
\end{tabular}} & \multicolumn{5}{|c|}{ Ameaças } & \multicolumn{5}{|c|}{ Oportunidades } \\
\hline & 0.05 & 0.09 & 0.18 & 0,36 & 0.72 & 0.72 & 0.36 & 0,18 & 0.09 & 0,05 \\
\hline 0,70 & 0.04 & 0.07 & 0.14 & 0.28 & 0.56 & 0,56 & 0.28 & 0,14 & 0.07 & 0,04 \\
\hline 0,50 & 0.03 & 0.05 & 0.10 & 0.20 & 0,40 & 0,40 & 0.20 & 0,10 & 0.05 & 0,03 \\
\hline 0,30 & 0,02 & 0,03 & 0,06 & 0,12 & 0.24 & 0,24 & 0,12 & 0,06 & 0,03 & 0,02 \\
\hline \multirow[t]{2}{*}{0,10} & 0,01 & 0,01 & 0,02 & 0,04 & 0,08 & 0,08 & 0.04 & 0,02 & 0.01 & 0,01 \\
\hline & 0,05 & 0,10 & 0,20 & 0,40 & 0,80 & 0,80 & 0,40 & 0,20 & 0,10 & 0,05 \\
\hline
\end{tabular}

Figura 2: Artefato 2.3: Matriz de probabilidade e impacto

Os jogadores poderão ganhar artefatos ao percorrer o tabuleiro do jogo. Algumas atividades práticas do jogo exigem que o jogador tenha o artefato correspondente a prática para ganhar os pontos, caso não o tenha, não poderá realizar a atividade. Por sua vez, os artefatos adquiridos podem ser uma ferramenta ou uma técnica. No jogo, caso o jogador alcance novamente uma casa que premia como um artefato que já o tenha, o jogador irá receber apenas os pontos de experiência equivalente.

O jogo não tem um final previsto, sendo assim, é necessário acordar o tempo de jogo antes de jogar. Após o tempo definido ser esgotado, vence quem acumular o maior número de pontos de experiência ao final do jogo [12]. Mais detalhes do jogo podem ser obtidos em http://bit.ly/2Sfgreh.

\section{Avaliação de Jogos Educativos}

O uso de jogos educacionais como meio de ensino está cada vez mais crescente $[25,24,14]$, porém de certa forma tem suas limitações, pois segundo Wangenheim e Shull [14] é importante que se tenha evidências dos benefícios dos jogos antes de sua utilização no ensino aprendizagem em sala de aula. Paludo e Raabe [9] salientam que a grande maioria dos jogos que são voltados para área de educação de computação não possuem avaliações significativas para verificar a validade do uso do jogo, e nem sempre apresentam resultados de efetiva aprendizagem com o uso dos mesmos.

Existem vários jogos que não possuem detalhamento suficiente sobre os efeitos de aprendizagem. Os jogos educacionais são promissores na educação de Engenharia de Software, porém existe uma deficiência para adotar mais o uso de jogos dentro de sala por não se ter evidências sobre as vantagens na aprendizagem dos alunos [14]. 
Para a avaliação rigorosa dos jogos são utilizados diversas metodologias que buscam avaliar níveis de aprendizagem, experiência do usuário e principalmente utilizar dos conceitos da taxonomia dos objetivos educacionais de Bloom. Entretanto, para Petri et al. [16], entre os modelos mais utilizados para a avaliação de jogos educacionais, está o modelo MEEGA+ [16].

O jogo The Risk Analysis Theory [12] foi avaliado considerando uma avaliação de percepção de uso dos participantes. No entanto, a avaliação contou com uma amostra muito pequena, sendo de apenas 3 (três) participantes. Além disso, a avaliação aplicada, apesar de ter seguido o método científico, não considerou as dimensões observadas pelo modelo MEEGA+ [16]. Sendo assim, percebeu-se a oportunidade de aplicar o modelo MEEGA+ para avaliar o jogo de Lamin [12]. A Seção a seguir apresenta o Modelo de Avaliação MEEGA+.

\subsection{O Modelo de Avaliação MEEGA+}

O modelo de avaliação MEEGA+ (Model for the Evaluation of Educational Games) é uma evolução do modelo MEEGA [1] para a obtenção de avaliação de qualidade de jogos digitais e não digitais [16]. O objetivo do modelo MEEGA+ consiste em "analisar jogos educacionais com o propósito de avaliar a percepção da qualidade em termos de experiência do jogador e percepção da aprendizagem do ponto de vista de alunos e instrutores no contexto de cursos superiores da área de computação.” [16]. Com base nesta definição, o modelo MEEGA+ tem o objetivo de aferir a qualidade dos jogos educacionais propondo fatores de qualidade para realização da avaliação [26]. O MEEGA+ é um modelo desenvolvido para avaliação de jogos educacionais por meio da aplicação de um questionário padronizado, usando-se a abordagem GQM (Goal/Question/Metric) para a definição dos resultados que envolvem as seguintes variáveis: motivação, experiência do usuário e aprendizagem do ponto de vista do aluno. Além disso, o modelo disponibiliza templates de questionários e planilhas para análise dos dados coletados [27].

Como metodologia, a avaliação do MEEGA+ avalia a experiência composta por um conjunto de dimensões: atenção focada, diversão, desafio, interação social, confiança, relevância, satisfação e usabilidade. O fator de usabilidade está dividido em outras cinco subdimensões: aprendizibilidade, operabilidade, estética, acessibilidade e proteção de erro do usuário. $O$ fator de aprendizagem percebida é subdividido em duas dimensões, a avaliação de curto prazo e o objetivo de aprendizagem. A avaliação de curto prazo objetiva avaliar o efeito global do jogo sobre a aprendizagem dos alunos. O objetivo de aprendizagem é personalizado, pois leva em consideração os objetivos de aprendizagem de cada jogo, tais como: análise, avaliação e criação $[16,28]$.

O MEEGA+ pode ser realizado de forma rápida e não intrusiva. Após a aplicação do jogo, é aplicado um questionário aos alunos para a coleta de dados buscando a qualidade percebida pelo aluno. A parte inicial do questionário coleta informações demográficas para identificar o perfil dos alunos e fornece duas versões (jogo digital e jogo não-digital) de um questionário de avaliação que pode ser adaptado de acordo com os objetivos de aprendizagem do jogo educacional e uma planilha de análise dos dados [28].

\section{Avaliação do The Risk Analysis Theory}

Esta Seção apresenta a avaliação do jogo The Risk Analysis Theory [12], aplicado em uma turma do curso de Sistemas de Informação na disciplina de Gerência de Projetos. O objetivo da avaliação foi de obter e atender os vieses anteriormente destacados em avaliar o jogo em questão utilizando o modelo MEEGA+ [16]. O estudo buscou avaliar a percepção do uso do jogo na disciplina e sua eficácia como ferramenta de ensino. As subseções a seguir apresentam o planejamento, execução e resultados da avaliação do jogo.

\subsection{Planejamento}

Para realizar o planejamento da avaliação, optou-se em seguir as recomendações realizadas em Travassos et al. [29], mesmo que este estudo seja classificado com um quase-experimento, pois a distribuição do indivíduos não foi realizada aleatoriamente, mas houve múltiplas observações [31]. Para avaliação do jogo foi escolhido o modelo de avaliação de jogos educativos MEEGA+. Como primeiro passo, o questionário modelo do MEEGA+ para jogos não digitais foi customizado de forma a avaliar também a aprendizagem dos estudantes em relação ao escopo da área de conhecimento de gerenciamento de riscos, que é elencado no The Risk Analysis Theory. Após esta etapa, o questionário do modelo MEEGA+ foi impresso para facilitar o preenchimento dos participantes. Os demais ativos do processo como plano de aula, material de aula e o próprio jogo, foram organizados pelo professor da disciplina. Aproveitou-se também o momento para realizar a avaliação original de Lamin [12], onde além de avaliar o jogo considerando as perspectivas do modelo MEEGA+ também permitiu avaliar os níveis de conhecimento. Sendo assim, foram impressos também os pré-testes e pós-testes. A seção a seguir detalha a execução da avaliação.

\subsection{Execução}

A execução do estudo exploratório foi realizada no segundo semestre de 2018 com o jogo The Risk Analysis Theory aplicado em uma turma de 22 alunos do ensino superior na disciplina de Gerência de Projetos. A execução da avaliação foi acompanhada pelo professor e orientador deste trabalho. Ao todo, foram necessários 2 encontros de 04 horas-aula para a execução da avaliação. Em um primeiro momento (realizado em 07 de novembro de 2018), todos os participantes foram submetidos a uma aula sobre o tema, onde o professor da disciplina apresentou o planejamento para o encontro conforme detalhado na Tabela 1.

Tabela 1: Plano de Aula

\begin{tabular}{|c|c|c|c|}
\hline Agenda & Conteúdo & $\begin{array}{c}\text { Objetivos da } \\
\text { Aprendizagem }\end{array}$ & Estratégia \\
\hline 04h/aula & $\begin{array}{l}\text { Gerenciamento } \\
\text { dos Riscos }\end{array}$ & $\begin{array}{l}\text { - Planejar o } \\
\text { gerenciamento de riscos; } \\
\text { - Identificar riscos em } \\
\text { projetos; } \\
\text { - Realizar análise } \\
\text { qualitativa e } \\
\text { quantitativa de riscos; } \\
\text { - Planejar respostas aos } \\
\text { riscos; } \\
\text { - Monitorar os riscos. }\end{array}$ & $\begin{array}{l}\text { - Aulas } \\
\text { expositivas } \\
\text { dialogadas; } \\
\text { - Atividade } \\
\text { prática; }\end{array}$ \\
\hline
\end{tabular}


Logo após a aula, os participantes foram convidados a participar do experimento, onde foram orientados pelo professor no preenchimento do termo de consentimento para participação do estudo. Todos os participantes realizaram a avaliação de préteste contendo 20 questões objetivas sobre Gerenciamento de Risco. Cabe ressaltar que o pós-teste também contemplou 20 questões objetivas, onde ambas as provas foram balanceadas considerando os níveis cognitivos da taxonomia de Bloom. Para tanto, foram utilizadas as avaliações objetivas elaboradas no trabalho de Lamin [12], que devido à baixa amostra para o experimento, a autora optou na ocasião em não realizar a sua aplicação. Os questionários de pré-teste e pós-teste utilizados nesta avaliação podem ser acessados em http://bit.ly/2RxkKkC.

No encontro seguinte (realizado no dia 14 de novembro de 2018), a turma foi dividida em três grupos de 7 (sete) participantes cada, como pode ser observado na Figura 3. Antes do início de cada partida, os participantes foram orientados sobre as regras do jogo pelo professor da disciplina. O primeiro grupo executou o jogo enquanto os demais integrantes dos outros grupos ficaram em outra sala aguardando. Após o primeiro grupo terminar a partida, o segundo grupo iniciou a partida do jogo e em seguida, o terceiro grupo. Ao finalizar cada rodada do jogo, os participantes realizavam o preenchimento do pós-teste $\mathrm{e}$ do questionário do modelo MEEGA+.

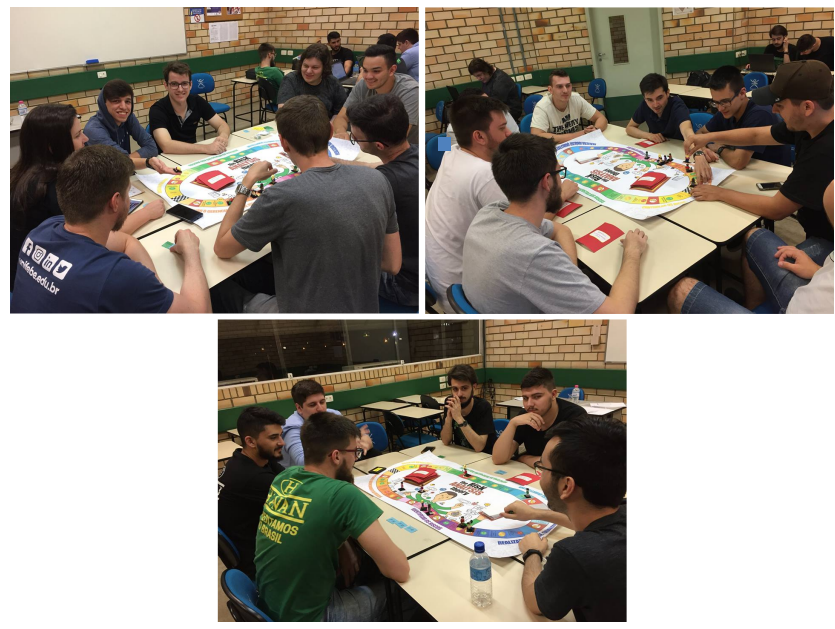

Figura 3: Aplicação do jogo na disciplina de Gerência de Projetos.

Após a execução desses passos, os documentos foram recolhidos e os dados foram passados para a planilha de análise do modelo. A seção a seguir apresenta o resultado das avaliações.

\section{Resultado da Avaliação}

Esta seção apresenta os resultados das avaliações aplicadas. Como destacado na seção anterior, foram realizadas duas avaliações. A primeira avaliação objetivou a verificação dos níveis de conhecimento dos participantes, permitindo analisar se houve aumento do conhecimento após a intervenção do jogo como método de complemento no ensino. A segunda avaliação, teve como objetivo avaliar o jogo utilizando um modelo de avaliação de jogos educacionais. Assim, as subseções a seguir detalham o resultado de cada avaliação.

\subsection{Avaliação dos Níveis de Aprendizagem}

Esta seção apresenta os resultados obtidos na avaliação de níveis de aprendizagem, conforme retrata a Figura 4. Como pode ser observado, 10 (dez) participantes (45,45\% da amostra) do total de 22, obtiveram notas maiores no pós-teste, demonstrando aumento de nível de conhecimento nestes participantes sobre o assunto abordado. No entanto, 8 (oito) participantes (correspondente a 36,36\% da amostra) obtiveram resultados inferiores aos obtidos no pré-teste. O restante da amostra, sendo $13,64 \%$ (3 participantes) obtiveram o mesmo resultado nas duas avaliações. Estes dois últimos resultados talvez possam ser justificados pelo esforço necessário dos participantes em realizar as avaliações. Os autores deste trabalho acreditam que a quantidade de questões possa ter contribuído para o desgaste dos participantes. Por outro lado, os autores deste estudo também concordam que uma quantidade pequena de questões não contribuiria para um resultado no qual pudesse expressar uma dedução valida sobre o resultado da avaliação. Outro fator que merece ser destacado, é quanto ao tempo entre a aplicação do pré-teste e do pós-teste, o intervalo de 7 (sete) dias entre as avaliações poderia ter afetado no resultado, visto que a primeira avaliação foi realizada imediatamente após a apresentação do conteúdo.

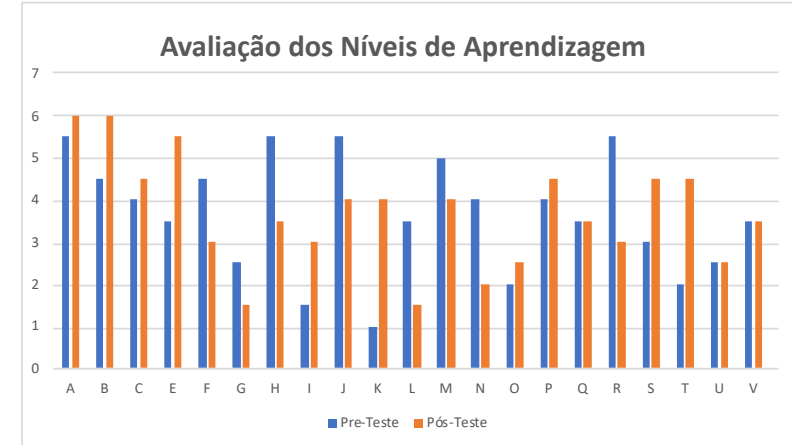

Figura 4: Resultado da Avaliação dos Níveis de Aprendizagem

Sendo assim, a partir dos resultados observados, não é possível afirmar que o jogo promoveu alguma melhora nos níveis de aprendizagem dos participantes, sendo necessário a realização de mais avaliações.

\subsection{Resultado Avaliação com Modelo MEEGA+}

Esta seção apresenta o resultado da avaliação do jogo através do instrumento de medida (questionário) do modelo MEEGA+. O questionário é dividido em 3 seções: (i) Informações demográficas; Experiência do jogador; e, Percepção da aprendizagem. Em relação as informações demográficas, a amostra é dominante de integrantes do gênero masculino, composta por $95 \%$ da amostra. A faixa etária da maioria $(91 \%$ da amostra) dos participantes é de 18 a 28 anos. Questionados sobre a frequência na qual costuma jogar jogos digitais, observa-se que os jogos estão presentes no cotidiano dos participantes, dos quais, 32\% responderam jogar todos os dias, e a mesma porcentagem respondeu jogar raramente. Os demais participantes responderam jogam semanalmente $(23 \%$ da amostra) ou costumam jogar mensamente $(13 \%$ dos 
XI Computer on the Beach

participantes). Quanto à utilização de jogos não digitais, a maioria dos participantes respondeu joga raramente este tipo de jogo (68\% da amostra), os demais disseram jogar pelo menos uma vez por mês (18\%), e $14 \%$ dos participantes jogam pelo menos uma vez por semana.

$\mathrm{O}$ aspecto de qualidade de experiência do jogador é percebida em termos de fatores e dimensões de qualidade. $\mathrm{O}$ conjunto de dimensões medido são: atenção focalizada, diversão, desafio, interação social, confiança, relevância, satisfação, usabilidade e aprendizado percebido. A Figura 5 mostra o resultado da avaliação em cada uma das dimensões.

Ao observar o resultado da avaliação da experiência do jogador, identificou-se que o jogo proporcionou uma experiência positiva aos participantes, destacando-se as dimensões de interação social, diversão e relevância. Este resultado é consoante com o observado por Petri et al. (2018) na avaliação de jogos não-digitais.

Diante do aspecto de usabilidade, o jogo obteve resultado positivo, destacando a facilidade de jogar, a clareza das regras e legibilidade do jogo quanto as fontes utilizadas. No entanto, outras questões como "eu precisei aprender poucas coisas para poder começar a jogar" e "as cores utilizadas no jogo são compreensíveis" obtiveram avaliação neutra dos participantes. Observa-se que, para os participantes, o jogo não agradou em relação a combinação entre os elementos de textos, cores e fontes, não sendo consistentes.

A avaliação da confiança do jogador foi positiva, indicando que as regras do jogo estavam bem elaboradas e foram de fácil compreensão dos participantes. No entanto, metade dos participantes se sentiram indiferentes em relação a sua impressão ao olhar o jogo pela primeira vez, e também, ao quanto o jogo seria fácil de jogar.

O jogo também foi consideram adequado quanto o desafio, apresentando desafios adequados e novos desafios com ritmo adequado, sem se tornar monótono em suas tarefas.

Os participantes afirmaram se sentirem satisfeitos com o método que foi utilizado para o reforço no ensino, sendo que a grande maioria afirmaram que recomendaria o jogo para seus colegas. No entanto, alguns participantes, mesmo que poucos, relataram que o avanço no jogo não dependeu de seu esforço pessoal.

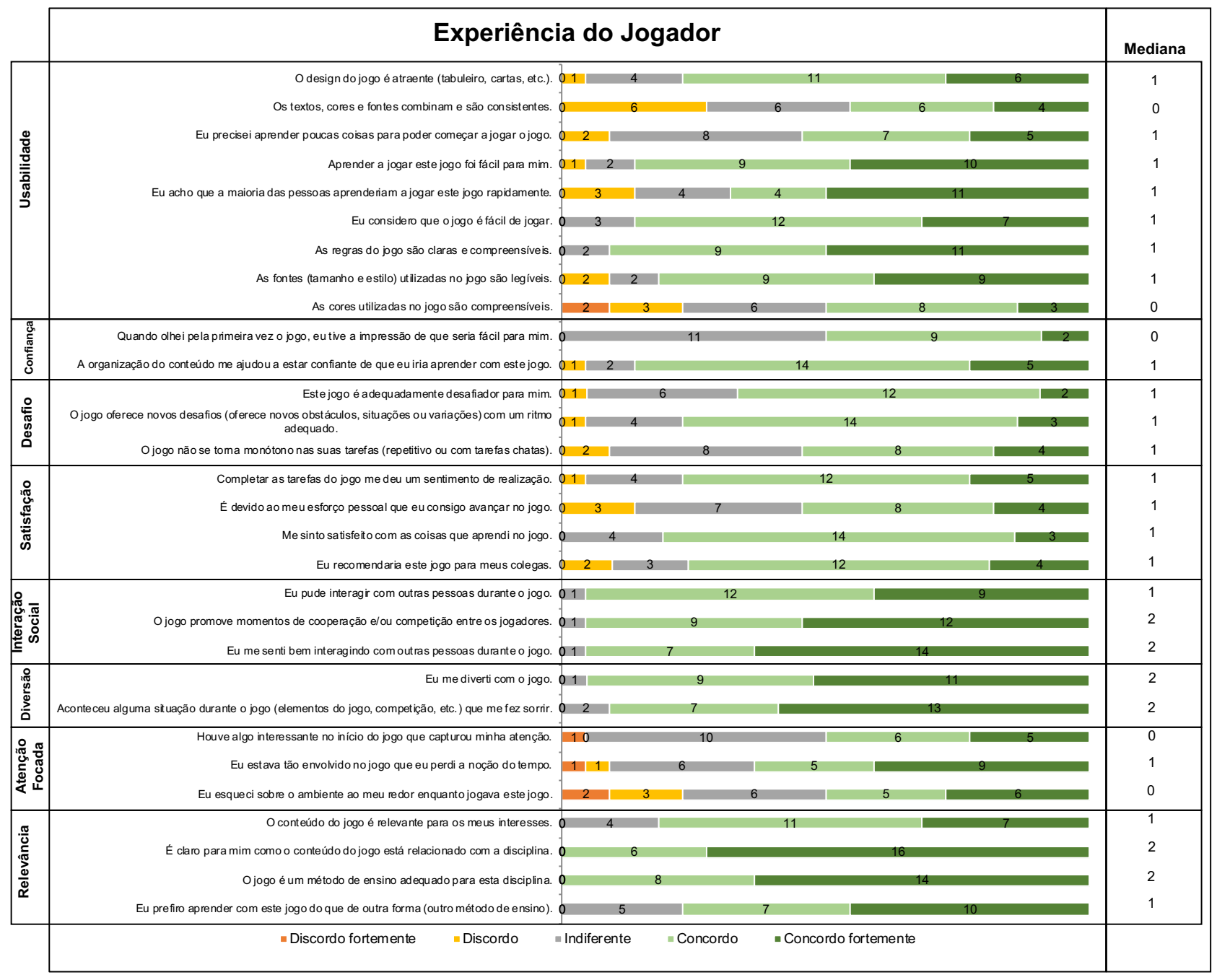

Figura 5. Avaliação da experiência do jogador 
A interação social e diversão foram os aspectos mais bem pontuados pelos participantes. Isso indica que o jogo prestou o papel de proporcionar diversão enquanto interagia com os demais participantes. O semblante dos participantes permite observar esse comportamento durante a aplicação do jogo, conforme pode ser observado na Figura 3.

Observando a atenção focada aplicada ao jogo pelo jogador, foi o aspecto que mais apresentou avaliações neutras e de não concordância, embora avaliada positivamente. Petri et al. [30] explicam que este resultado é normal devido a natureza dos jogos não-digitais, sendo necessário explorar outras dimensões dos jogos (como desafio, interação social e diversão) para obter uma maior imersão dos jogadores.

Por fim, o aspecto relevância também foi avaliado positivamente. Os participantes, na sua maioria, concordaram plenamente que o conteúdo do jogo está relacionado com a disciplina, e que ele é adequado como método de ensino. Além disso, os participantes também afirmaram preferir aprender com o jogo em vez de outra forma ou método de ensino.

Além de realizar a avaliação da experiência do jogo, o modelo MEEGA+ [16], avalia fatores de qualidade de percepção de aprendizagem em duas dimensões (resultados retratados na Figura 6). A primeira dimensão realiza a avaliação de aprendizagem de curto prazo objetivando avaliar o efeito geral do jogo sobre a aprendizagem dos participantes. A primeira questão na avaliação de curto prazo, questiona o participante se o jogo contribuiu em sua aprendizagem na disciplina. Somente dois participantes afirmaram serem indiferentes a intervenção do jogo, os demais concordam que o jogo contribuiu em sua aprendizagem. A segunda questão, questiona se o participante considera o jogo eficiente em sua aprendizagem em relação a outras abordagens. Aqui, novamente dois participantes se sentiram indiferentes em relação a intervenção, os demais concordam que o jogo é mais eficiente em relação a outras abordagens.

A segunda dimensão da percepção de aprendizagem avalia os objetivos de aprendizagem. As questões desta dimensão são personalizadas, pois devem considerar os objetivos de aprendizagem de cada jogo [16]. Assim, foram incluídas três questões relacionadas à disciplina focada no gerenciamento de riscos. A primeira questão obteve como resultado a concordância de todos os participantes, onde foram perguntados se "O jogo apresentou o conteúdo de Gerência de Riscos de forma organizada e de fácil entendimento durante o jogo”. A segunda questão visou verificar a percepção dos participantes quanto a sua contribuição no aumento de seu interesse sobre a área de gerenciamento de risco. A maioria dos participantes concordou em ter aumentado seu interesse na área, mesmo sendo em graus diferentes de concordância, além disso, foi a questão que mais apresentou neutralidade e discordância. A última questão objetivou verificar com os participantes a sua concordância em relação a ajuda que o jogo proporcionou no entendimento das atividades de gerenciamento de risco. A grande maioria concordou que o jogo permitiu conhecer melhor as atividades realizadas no gerenciamento de risco.

Sendo assim, o resultado da avaliação indica uma boa aceitação do jogo como ferramenta de apoio ao ensino no assunto de gerenciamento de risco. Alguns pontos de melhoria do jogo podem ser observados com o resultado, a citar: as cores do jogo; e, na mecânica do jogo para torná-lo mais desafiador. Além disso, a diversão e interação social foram avaliados como os pontos mais fortes do jogo. Em relação à percepção de aprendizagem, os participantes avaliaram de forma positiva, sendo indício de que o jogo é uma ferramenta atraente e que promove a aprendizagem.

\subsection{Discussão dos Resultados}

Duas avaliações foram realizadas para verificar se existe benefícios na utilização do jogo The Risk Analysis Theory como ferramenta para o apoio ao ensino.

A partir dos resultados da avaliação dos níveis de conhecimento, não foi possível obter indícios de que o jogo promoveu aumento de conhecimento dos participantes. Pois, a diferença entre as avaliações de pré-teste e pós-teste foi muito pequena, não permitindo afirmar se realmente a intervenção utilizando o jogo realmente promoveu aprendizagem.

A avaliação do jogo utilizando o modelo MEEGA+ permitiu verificar a experiência do jogador e sua percepção de aprendizagem. O jogo obteve um resultado positivo quanto à experiência do jogador na maioria das dimensões observadas pelo modelo. Os pontos fracos ficaram em relação à dimensão de usabilidade e atenção focada. Quanto ao aspecto de usabilidade as indicações de melhorias estão relacionadas as cores do jogo, tendo em vista que alguns participantes relataram se confundirem um pouco por haver alternância nas cores do tabuleiro. Em relação à dimensão de atenção focada, a baixa concordância e neutralidade podem ter ocorrido devido o jogo ter um pilar de sustentação baseado em "sorte", o que não exige muito foco ou esforço em construção de estratégias elaboradas para se ganhar. Em relação à percepção de aprendizagem, o resultado foi positivo para o jogo, onde os participantes afirmaram que o jogo contribuiu para sua aprendizagem e consideraram uma ferramenta eficiente para isso, além de despertaram interesse pela área estudada e nos entendimentos das atividades realizadas.

O resultado da avaliação a partir do modelo MEEGA+ indica que o jogo contribui positivamente no aprendizado dos estudantes. As recomendações dos participantes podem ser adotadas para melhorar a experiência dos jogadores. Com a aplicação do experimento, percebeu-se de que o jogo proporcionou uma ótima interação dos participantes com bastante dinâmica, diversão e competição. Além disso, outro aspecto de relevância a ser destacado é de que os participantes concordam que o jogo apresenta benefícios como ferramenta de ensino para a disciplina de gerenciamento de risco.

\subsection{Validade dos Resultados}

Travassos et al. [29] contribui em destacar a importância a respeito dos resultados do experimento quanto a sua validade. Sendo assim, as seguintes ameaças foram identificadas e tratadas: (i) validade de conclusão: relativa ao relacionamento entre o tratamento e o resultado do experimento. O modelo adotado foi elaborado sistematicamente, sendo validado os formulários para entrada de dados e aplicação de método estatístico para expressar os resultados. Quanto as avaliações de pré-teste e póstestes, tentou-se minimizar esta ameaça utilizado questões niveladas pela taxonomia de Bloom, no entanto, nenhum método estatístico foi aplicado ao resultados destas avaliações; (ii) validade de construção: relativa a teoria e a observação do estudo, ou seja, o relacionamento com influência dos tratamentos nos resultados observados. O método selecionado para avaliação 
deste estudo descarta essa ameaça, pois o modelo escolhido apresenta várias contribuições na literatura sendo amplamente conhecido; (iii) validade interna: relativo as influências de casualidade, na qual o pesquisador não possui conhecimento ou controle. Para aumentar a validade desta variável, optou-se em dividir o experimento em dois momentos para não fadigar os participantes devido ao tempo de exposição à intervenção. Também observou-se a alocação dos participantes em salas diferentes para acomodar os indivíduos que aguardariam a intervenção ser realizada com os outros grupos. Para representação da população, optou-se pela escolha de indivíduos que estivesse na mesma turma da disciplina de Gerência de Projetos. Por fim, todos os participantes foram submetidos a mesma intervenção; e, (iv) validade externa: relativo a habilidade de generalizar os resultados. Como descrito na validade interna os participantes podem ser considerados uma população representativa de indivíduos com o mesmo conhecimento teórico sobre gerenciamento de riscos.

\section{Conclusão}

Este trabalho apresentou o resultado de duas avaliações distintas no jogo de tabuleiro educacional The Risk Analysis Theory [12]. O jogo foi aplicado no reforço da aprendizagem na disciplina de Gerência de Projeto em uma instituição de ensino superior em Santa Catarina. A partir do resultado da primeira avaliação não foi possível verificar se o jogo promoveu aumento nos níveis de aprendizagem dos participantes. A segunda avaliação, utilizando o modelo MEEGA+ [16], indicou que o jogo contribui positivamente para o aprendizado dos estudantes. No entanto, alguns pontos de melhorias para o jogo foram identificados. Como trabalho futuro, destaca-se a realização de mais rodadas na avaliação dos níveis de aprendizagem e na percepção da qualidade do jogo. Destaca-se também a aplicação de método estatístico adequado para verificação dos níveis de conhecimento.

\section{AGRADECIMENTOS}

Os autores agradecem aos alunos do Curso de Sistemas de Informação do Centro Universitário de Brusque - UNIFEBE, pela participação na avaliação.

\section{REFERÊNCIAS}

[1] Savi, R., (2011). Avaliação de Jogos Voltados Para a Disseminação do Conhecimento, p. 236. Tese (Universidade Federal de Santa Catarina Programa de Pós-Graduação em Engenharia e Gestão do Conhecimento, Florianópolis, SC)

[2] Swebok, 2004. Guide to the So ware Engineering Body of Knowledge. A project of the IEEE Computer Society Professional Practices Committte, IEEE Computer Society.

[3] Pressman, R. S. (2011) Engenharia de So ware: uma abordagem profissional. 7. ed. São Paulo: Pearson Makron Books.

[4] Mendes, F. F; Oliveira, J. L.; Fernandes, P. G.; Souza, A. S., (2007). Análise de Riscos na Implantação de Melhorias de Processos de So ttvare. Pro ality (UFLA) , v. 3, p. 25-31.

[5] Silva, A. C., (2010). Jogo educacional para apoiar o ensino de técnicas para elaboração de testes de unidade, Dissertação (Universidade do Vale do Itajaí de Santa Catarina, Curso de Mestrado Acadêmico em Computação Aplicada, Florianópolis.

[6] Wangenheim, C. G., SILVA, D. A., (2009). al Conhecimento de So ware é Importante para um Profissional de So ttware?. Fórum de Educação em Engenharia de So ttware (FEES).

[7] Huang, S; Distante, D. (2006) On Practice-Oriented So ware Engineering Education., CSEETW '06 Proceedings of the 19th Conference on So ttvare Engineering Education and Training Workshops.
[8] Gramigna, M. R. M. (1993) Jogos de Empresa. São Paulo: Makron Books.

[9] Paludo, L; Raabe, A. (2010). Análise de Jogos Educativos de Computador para Gerência de Projetos de So ttware. XVIII Workshop sobre Educação em Computação (WEI), Belo Horizonte, Minas Gerais.

[10] Fernandes, L; Werner, C. M. L., (2009). Sobre o uso de Jogos Digitais para o Ensino de Engenharia de So ware. "BDBComp - Biblioteca Digita Brasileira de Computação". [Online].

[11] Dantas, A. R., (2003). Jogos de Simulação no Treinamento de Gerentes de Projetos de So ttvare. Tese (Mestrado Engenharia de Sistemas e Computação) - Programa de Pós-Graduação em Engenharia de Sistemas e Computação, UFRJ, Rio de Janeiro, RJ - p.106.

[12] Lamim, J. C. (2017) Um Jogo de Apoio ao Ensino à Gerência de Riscos. Trabalho de Conclusão de Curso - Centro Universitário de Brusque UNIFEBE, Brusque - Santa Catarina.

[13] PMI. (2017) Um Guia do Conhecimento em Gerenciamento de Projetos: Guia PMBOK. 6. ed. Newtown Square: Project Management Institute.

[14] Wangeheim, C. G., SHULL, (2009) F. To Game or Not to Game? So ware, IEEE, 26(2), 92-94.

[15] All, A., Castellar, E. P. N., Looy, J. V. (2016) Assessing the effectiveness of digital game-based learning: Best practices. Computers \& Education, 92-93, 90-103.

[16] Petri, G; Wangenheim, C. G.; Borgatto, A. F. (2017). A Large-Scale Evaluation of a Model for the Evaluation of Games for Teaching So tware Engineering. IEEE/ACM 39th International Conference on Software Engineering: Software Engineering Education and Training Track (ICSESEET), Buenos Aires/Argentina.

[17] Jappur, R. F.; Spanhol, F. J.; Forcellini, F. A. (2017). Modelo conceitual para criação, aplicação e avaliação de jogos educativos digitais, RENOTE, vol. 14, no 2, jan. 2017.

[18] Savi, R.; Ulbricht, V. R. (2008). Jogos educacionais digitais: Benefícios e Desafios. RENOTE, vol. 6, no 1, jun. 2008.

[19] Ariffin, M. M; Oxley, A.; Sulaiman, S. (2013). Evaluating Game-Based Learning Effectiveness in Higher Education. Procedia-Social and Behavioral Sciences, v. 123, p. $20-27$.

[20] Brito, L. F.; Ramos, R. A.; Neto, R. F. O.; Leal, B. G. (2018). Analysis of the game SIMULES in support of teaching and learning So ttware Engineering. Brazilian Journal of Education, Technology and Society, vol. 11, no 4, p. 680 692

[21] Monsalve, E. S.; Werneck, V. B. B.; Leite, J. C. (2010). SimulES-W: Um Jogo para o Ensino de Engenharia de So ttware. Fórum de Educação em Engenharia de So ttware (FEES), Bahia.

[22] Meirelles, L., Peixoto D., Figueiredo, E., Resende, R., Pádua, C., Monsalve, E., Leite, J. C. S. P., WERNECK, V., (2011). Uso de Jogos para o Ensino de Engenharia de So ttware. CBSo tt2011 - SBES - FEES, São Paulo.

[23] Souza, M.; França, C. (2016). O Sucesso dos Jogos para Ensino de Disciplinas de Engenharia de So ttware sob a Ótica de uma Teoria Motivacional. Simpósio Brasileiro de Informática na Educação - SBIE, vol. 27, no 1, p. 450.

[24] Monsalve, E. S. \$014). Uma Abordagem para Transparência Pedagógica usando Aprendizagem Baseada em Jogos. Tese (Programa de Pós-graduação em Informática do Departamento de Informática do Centro Técnico Científico da PUC-RIO).

[25] Wangenheim, C. G., Wangenheim, A. (2012). Ensinando Computação com Jogos. Editora Bookess, Florianópolis.

[26] Moreira, G. G. Ł018). Avaliação da percepção de jogadores sobre jogos educacionais para ensino de SCRUM. Monografia (Graduação em Engenharia de So ttware) - Campus de Russas, Universidade Federal do Ceará, Russas.

[27] Petri, G., Wangenheim, C. G., Borgatto, A. F.. (2017) Evolução de um Modelo de Avaliação de Jogos para o Ensino de Computação. Anais do XXV Workshop sobre Educação em Computação (WEI), pp.2327-2336.

[28] Soares, R.; Petri, G.; Wangenheim, C. G.; Conte, T.; Marques, A. (2018) Assistant MEEGA+: Uma ferramenta de apoio para avaliação de jogos educacionais usando modelo MEEGA+. Simpósio Brasileiro de Informática na Educação, Fortaleza/CE.

[29] Travassos, G. H., Gurov, D., Amaral, E. A. G., (2002). Introdução à Engenharia de So ttware Experimental, Relatório Técnico RT-ES-590/02, Programa de Engenharia de Sistemas e Computação COPPE / UFRJ, Rio de Janeiro.

[30] Petri, G; Calderón, A; Wangenheim, C. G.; Borgatto, A. F.; Ruiz, M. (2018). Benefícios dos Jogos Não-Digitais no Ensino de Computação. In: Workshop Sobre Educação Em Computação (WEI_CSBC), 26. , 2018, 1/2018. $26^{\circ}$ Workshop sobre Educação em Computação (WEI 2018). Porto Alegre.

[31] Trochim, W. M., Donnelly, J. P.. (2006) e Research Methods Knowledge Base. 3rd Edition, Atomic Dog, Cincinnati, $\mathrm{OH}$. 